\title{
Article \\ RF Pogo-Pin Probe Card Design Aimed at Automated Millimeter-Wave Multi-Port Integrated-Circuit Testing
}

\author{
K. M. Lee ${ }^{1}$, J. H. Oh ${ }^{2}$, M. S. Kim ${ }^{2}$, T. S. Kim ${ }^{2}$ and M. Kim ${ }^{1, *}$ \\ 1 School of Electrical Engineering, Korea University, Seoul 02841, Korea \\ 2 Samsung Electronics Co., Ltd., Suwon 16677, Korea; jung_hoon.oh@samsung.com \\ * Correspondence: mkim@korea.ac.kr
}

check for updates

Citation: Lee, K.M.; Oh, J.H.; Kim, M.S.; Kim, T.S.; Kim, M. RF Pogo-Pin Probe Card Design Aimed at Automated Millimeter-Wave Multi-Port Integrated-Circuit Testing. Electronics 2021, 10, 2446. https:// doi.org/10.3390/electronics10192446

Academic Editor: Leonardo Pantoli

Received: 4 August 2021

Accepted: 5 October 2021

Published: 8 October 2021

Publisher's Note: MDPI stays neutral with regard to jurisdictional claims in published maps and institutional affiliations.

Copyright: (c) 2021 by the authors. Licensee MDPI, Basel, Switzerland. This article is an open access article distributed under the terms and conditions of the Creative Commons Attribution (CC BY) license (https:// creativecommons.org/licenses/by/ $4.0 /)$.

\begin{abstract}
A prototype RF probe card is assembled to test the feasibility of Pogo-pins as robust probe tips for the automized testing of multiple-port millimeter-wave circuits. A custom-made ceramic housing machined from a low-loss dielectric holds an array of 157 Pogo-pins, each with $2.9 \mathrm{~mm}$ length in fixed positions. The ceramic housing is then mounted onto a probe-card PCB for power-loss measurements on two signal-ground Pogo-pin connections arbitrarily selected from the array. The probing results on a test circuit with a simple thru-line indicate a successful power transfer with a small insertion loss of less than $0.5 \mathrm{~dB}$ per single Pogo-pin connection up to $25 \mathrm{GHz}$. A new probe card design using shorter Pogo-pins is being prepared to extend the operation frequency to beyond $40 \mathrm{GHz}$.
\end{abstract}

Keywords: RF probe cards; Pogo-pin probes; automized RF testing

\section{Introduction}

RF probes are critical components in high-frequency on-wafer testing environments. They are extensively used to deliver high-frequency signals to two-port integrated circuits that include $50-\Omega$ probe pads. Most RF probes typically contain microscopic landing tips attached to the end of a coaxial cable in the form of coplanar waveguide (CPW) transmission lines [1]. Commercial single-port probes are readily available from manufactures such as Cascades Inc. and GGB Inc. for frequencies reaching up to $1.1 \mathrm{THz}$. From our internal experiment, a 50-GHz probe from GGB (50A-GSG-100-P) is found to be almost loss-free with the measured insertion loss of less than $0.4 \mathrm{~dB}$ at $50 \mathrm{GHz}$.

Recent developments in integrated system on chips (SoC) such as multiple RF-channel beamforming systems require a large number of probe tips bundled together to form a high-density array for simultaneous signal delivery during automated on-wafer testing. RF probe cards offer testing convenience by mounting multiple probe tips on a single PCB board. Several examples of commercially available probe cards are listed in Table 1 along with the tip shape, material, and pitch size information. The multi-tip probe product from GGB [2] simply joins conventional single-port probes together to form an array. In other products, RF probe tips are aligned in GSSG or GSGSG configuration to form differential ports [3]. Similar to regular single-port RF probes, these types of probes require an extreme care to avoid tip damages. Another example shows the MEMS process being applied to fabricate arrays of needle tips with very small resistive losses using high-stiffness metals such as $\mathrm{BeCu}$, tungsten, and Titanium composites [4]. Initially, the fabrication process could only form probe tips in a linear array. Later, a new MEMS pyramid-shaped design of tips fabricated from a thin-film membrane was introduced by Cascades [5] to enable the formation of two-dimensional arrays. These probe tip arrays are still very expensive and suffer from durability issues. For these reasons, Pogo-pins using internal springs with improved resilience have been introduced for the RF probing applications. Yokowo demonstrated 250 pin array in a metallic housing to achieve an acceptable signal delivery 
for frequencies only up to $6 \mathrm{GHz}$ [6]. Pasternack presented delicate and miniscule 40$\mu \mathrm{m}$ diameter Pogo-pins as a single-port probe working up to $40 \mathrm{GHz}$ [7]. A test socket containing an array of 37 sturdier Pogo-pin elements with a 5-mm length and a $0.5-\mathrm{mm}$ diameter are introduced in [8]. In an unconventional setup, two traditional pico-probes are used to directly contact a signal-ground Pogo-pin pair on each end to measure $3 \mathrm{~dB}$ of loss at $20 \mathrm{GHz}$ [8]. In the latest Pogo-pin research, the performance of three Pogo-pins providing G-S-G connections for CPW transmission lines on two PCB's positioned at 90degree angle [9], and an extensive theoretical analysis using HFSS on a Pogo-pin array for insertion loss and crosstalk coupling in the millimeter-wave range [10] are discussed. In this paper, we present a fully assembled RF probe card that uses an array of 157 Pogo-pins packaged in a low-loss ceramic with the pitch size fixed by the millimeter-wave circuit under testing. In addition to actual experiments, we performed HFSS simulations for a detailed analysis of RF probe card performance. Our work uses oversized Pogo-pins with $2.9-\mathrm{mm}$ length and $0.2-\mathrm{mm}$ diameter in order to withstand a minimum of $100 \mathrm{k}$ landing count.

Table 1. List of commercially available multiple-port RF probes.

\begin{tabular}{cccccccc}
\hline Ref. & Manufacturer & Max. Frequency & No. Tips & Type & Material & Pitch & Insertion loss \\
\hline$[2]$ & GGB & $110 \mathrm{GHz}$ & 36 & Needle & BeCu/tungsten & $50 \mathrm{um}$ & $1 \mathrm{~dB} @ 50 \mathrm{GHz}$ \\
\hline$[3]$ & T-plus & $67 \mathrm{GHz}$ & 5 & Needle & Nickel alloy & $50 \mathrm{um}$ & $0.5 \mathrm{~dB} @ 25 \mathrm{GHz}$ \\
\hline$[4]$ & MPI & $6 \mathrm{GHz}$ & 15 & Needle & Titanium & $50 \mathrm{um}$ & $1 \mathrm{~dB} @ 6 \mathrm{GHz}$ \\
\hline$[5]$ & Cascade & $81 \mathrm{GHz}$ & 438 & Pyramid & Nickel alloy & $50 \mathrm{um}$ & - \\
\hline$[6]$ & Yokowo & $6 \mathrm{GHz}$ & 250 & Pogo-pin & Au/Pd Alloy & $130 \mathrm{um}$ & $1 \mathrm{~dB} @ 6 \mathrm{GHz}-$ \\
\hline$[7]$ & Pasternack & $40 \mathrm{GHz}$ & 3 & Pogo-pin & Gold & $800 \mathrm{um}$ & $0.5 \mathrm{~dB} @ 40 \mathrm{GHz}$ \\
\hline- & This work & $25 \mathrm{GHz}$ & 157 & Pogo-pin & BeCu alloy & $350 \mathrm{um}$ & $0.5 \mathrm{~dB} @ 25 \mathrm{GHz}$ \\
\hline
\end{tabular}

\section{Housing Ceramic Design for Pogo-pin Array}

The RF probe card discussed in this paper is aimed at the automated testing of a specific SoC chip, namely a 5G phased-array system currently under development by Samsung Electronics for operation frequencies of 29 and $39 \mathrm{GHz}$. As shown in Figure 1, the ball map of the SoC under examination has a total of 157 ball pads for $32 \mathrm{RF}$ signal connections along the edge and in the middle of the chip marked as red boxes, and 56 ground connections surrounding the signal pads denoted as grey boxes. The signal and ground pad locations are carefully arranged in order to prevent RF signal loss due to reflection or radiation. The ball pads are connected to microstrip lines inside the chip, and these transmission lines are expected to have termination impedances of $50 \Omega$. The remaining 69 ball pads are for DC biasing shown as green boxes and system control as blue boxes, and they are placed without consideration of RF signal propagation. The total chip size is $3.3 \times 5.6 \mathrm{~mm}^{2}$ while the pitch sizes are horizontally $0.26 \mathrm{~mm}$, vertically $0.23 \mathrm{~mm}$, and diagonally $0.35 \mathrm{~mm}$.

The Pogo-pin selected for our RF probe card system is shown in Figure 2a. It is custom manufactured with the part number GN855AMR-DGPC by Leeno Industrial Inc. who specializes in RF probe products [11]. The Pogo-pin consists of four parts: the crownshaped terminal made of unplated special alloy that grabs the soft ball pad on the IC, the plunger made of gold-plated hardened BeCu with a spherical tip contacting the PCB, the barrel made of gold-plated special alloy, and the internal a made of gold-plated Music wire. Gold plated metals reduce conductor losses while $\mathrm{BeCu}$ provides the stiffness required for the pin. The barrel diameter of $0.2 \mathrm{~mm}$ is chosen for $50-\Omega$ impedances for the transmission lines formed by one signal and two adjacent ground Pogo-pins. The G-S-G arrangement of the Pogo-pins can be modeled as a transmission line with an impedance of $54 \Omega$ and electrical length of 80 degrees at $25 \mathrm{GHz}$. Although smaller barrel length is preferred for 
high-frequency RF performance, our barrel length of $2.3 \mathrm{~mm}$ is expected to provide better structural integrity.

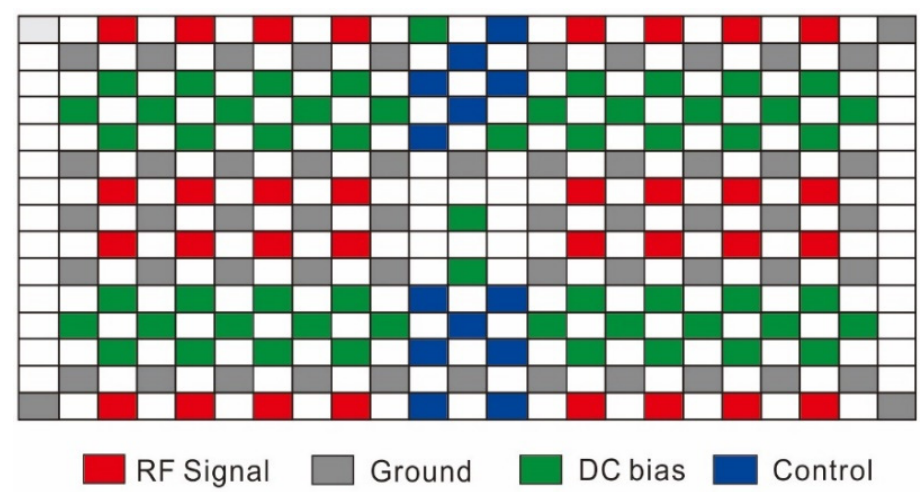

Figure 1. Ball pad map of the SoC under test with the chip size of $3.3 \times 5.6 \mathrm{~mm}^{2}$.

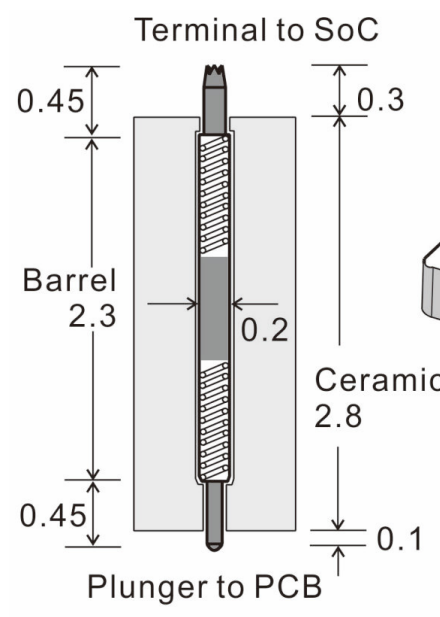

(a)

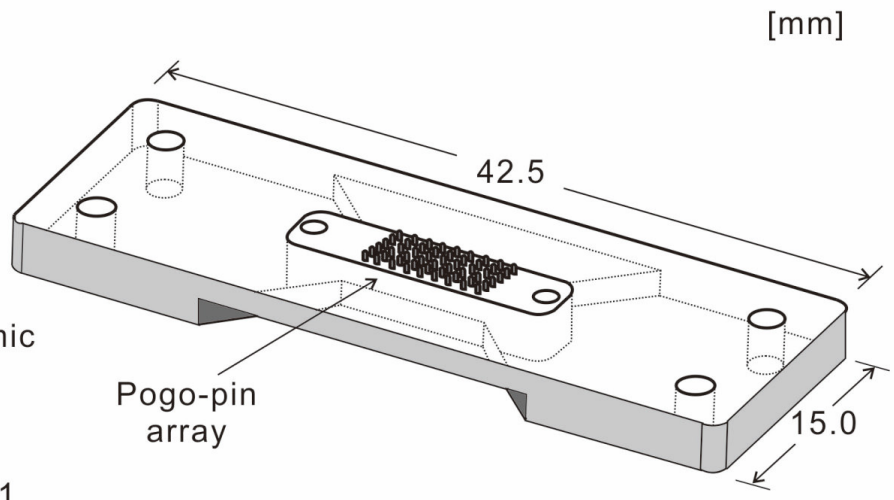

(b)

Figure 2. Sketches of (a) uncompressed Pogo-pin and (b) ceramic housing containing Pogo-pin array.

The ceramic housing is a key part for the RF probe card system along with the probecard PCB and RF connectors. The ceramic housing is custom-made to hold 157 pogo-pins for testing of the current version of the $5 \mathrm{G}$ phased array IC. It contains holes to fit the array of Pogo-pins aligned at required positions with an additional top cover to keep the pins in place as shown in Figure 2b. It is machined from MDS100 material with a dielectric constant of 3.34 and a loss tangent of 0.007. MDS100 material not only offers a firm grip on pogo-pins with small RF loss, but also ensures that the signal-to-ground separation distance of $0.35 \mathrm{~mm}$ provides a near $50-\Omega$ transmission-line impedance. In case a more tight pitch size is required, the ceramic housing could be fabricated from a low-loss metal/ceramic composite that offers a very high dielectric constant [12].

\section{Probe Card Measurements}

In order to analyze the performance of our RF probe card, the PCB substrate loss is estimated beforehand. A loss-estimation PCB that contains a 30-mm long straight transmission-line as shown in Figure 3 is separately prepared. This PCB, along with the main probe-card PCB, is fabricated from a 10 mil-thick Rogers R3003 substrate with a dielectric constant of 3. A backside-grounded CPW transmission line with a $50-\Omega$ characteristic impedance is formed using a ground-to-ground distance of $3 \mathrm{~mm}$ and a signal-line width of $0.6 \mathrm{~mm}$. Additional vias thru the PCB connect the front and back sides of the ground plane at a short interval to prevent any substrate resonances. A $50-\mathrm{GHz}$ network analyzer, Keysight E8364B, is used to obtain S-parameters, and the measured 
results show better than $1.3 \mathrm{~dB}$ of loss at $40 \mathrm{GHz}$. There are no resonances observed in the frequency response and the reflection loss stayed below $10 \mathrm{~dB}$ for all frequencies. RF connectors placed at the edge of the PCB are critical for preventing unexpected losses for millimeter-wave operation. We used 2.4-mm coaxial connectors, H2.4-LR-SR2, from Hirose Electric Co. that is well-suited for CPW line connections as the signal and ground pins are aligned on the same plane. The PCB substrate is pressed on to the connector pins using a pair of screws thru the PCB and the ground pins. It is also observed that there should not be any gaps between the PCB and connectors. The outer conductor of the RF connector and the PCB ground plane are soldered together. The simulated data with and without the RF connectors are also plotted in Figure 3 as dashed lines with a loss tangent value set to 0.007 to fit the measured data up to $25 \mathrm{GHz}$. Additional reflection loss can be prevented as long as the RF connectors are properly attached to the PCB.

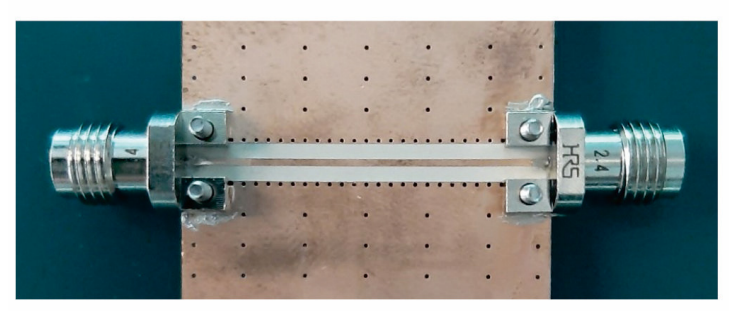

(a)

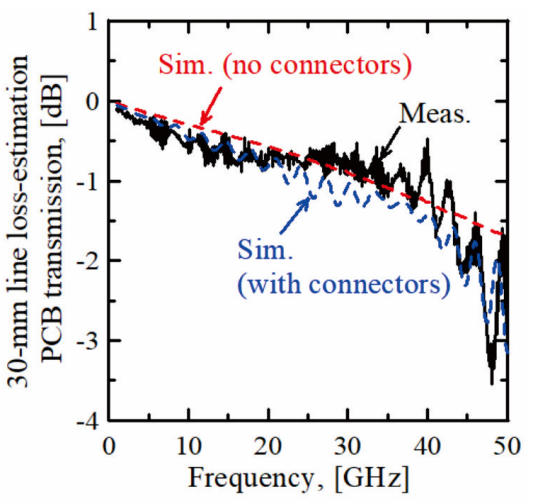

(b)

Figure 3. (a) Loss-estimation PCB and (b) the measured and simulated losses.

The RF probe card is constructed by bolting the ceramic housing onto the probe-card PCB with its sketch shown in Figure 4 and photograph in Figure 5a. The PCB is later mounted to a three-axis manipulator for the accurate positioning of the Pogo-pins on the test circuit. Although the ceramic housing contains a total of 32 RF signal Pogo-pins, the performance of a two-port RF signal connection is tested in the current experiment as the first step toward designing a full RF probe card system. Only two of the RF signal pogo-pins shown as red dots are connected to the CPW signal lines, and the remaining 155 pins with different colors are grounded or left unconnected.

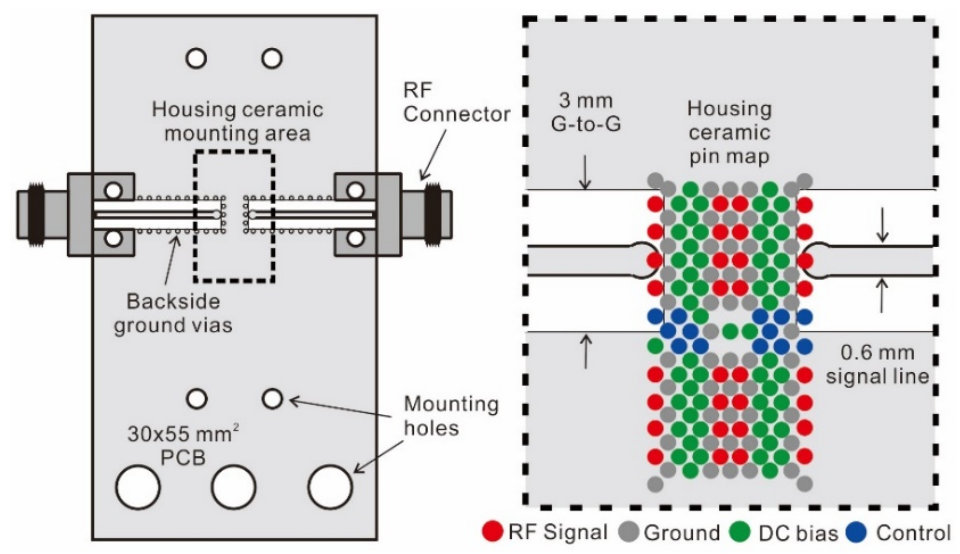

Figure 4. Sketch of probe-card PCB.

A test PCB containing a $50-\Omega$ CPW line forming a detour circle is fabricated using the same Rogers R3003 substrate, and its photograph is shown in Figure 5b. The full two-port insertion loss can be measured by placing the pogo-pins on the test PCB resulting in the 
total signal path length of $79 \mathrm{~mm}$. The pin landing area of the test PCB is identical to the probe-card PCB. Measurements show linearly increasing loss up to $25 \mathrm{GHz}$ where the insertion loss of $3.4 \mathrm{~dB}$ is measured as shown in Figure 5c. HFSS simulation results using the previously estimated PCB loss tangent of 0.007 indicate that the extra loss introduced by the two Pogo-pin connections is less than $1 \mathrm{~dB}$ up to $25 \mathrm{GHz}$. The reflection loss in Figure $5 \mathrm{~d}$ remains under $-10 \mathrm{~dB}$ up to $25 \mathrm{GHz}$.

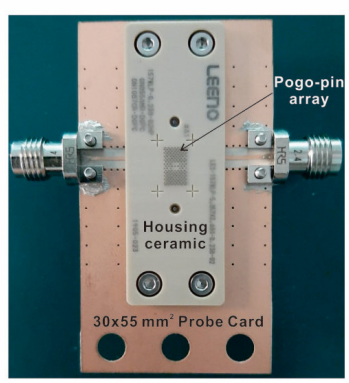

(a)

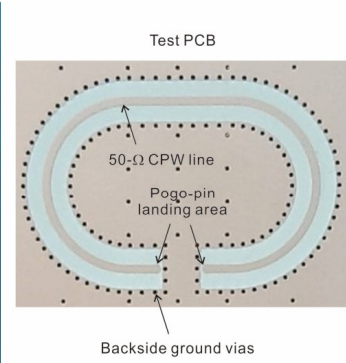

(b)

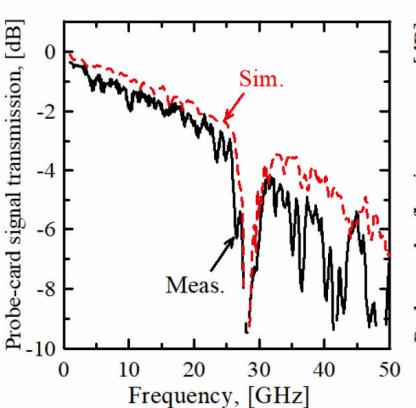

(c)

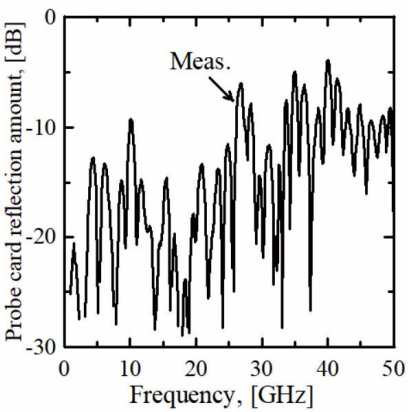

(d)

Figure 5. Photographs of (a) the probe card and (b) the test PCB, and (c) the total insertion and (d) reflection losses.

Other common tests that validate the RF probe card performance include inspections on the time domain-reflectometer (TDR) waveforms and the eye-diagrams with modulated input signals. TDR waveforms can be directly measured using a network analyzer or converted from the measured S-parameters using circuit simulators, such as Advanced Design Systems (ADS). The TDR waveform converted from the measured S-parameters of up to $50 \mathrm{GHz}$ for the probe card testing is plotted in Figure 6a. It shows the characteristic impedances of each segment of the two-port RF probe card system when one port is open circuited. In the time-domain conversion, the rise time of the excitation signal is set to $20 \mathrm{ps}$ to match the 50-GHz S-parameter limit. The measured waveform shows small deviations from $50 \Omega$ for the signal path of the probe card system with a slight delay added due to extra the RF connectors. Two impedance deviations of $54 \Omega$ occurring at 0.3 ps and 0.6 ps correspond to the Pogo-pin sections. Slightly larger impedances of 55 to 60 are observed for the RF connectors at $0.2 \mathrm{ps}$ and $0.8 \mathrm{ps}$. The second examination is performed by connecting a 34-GHz arbitrary waveform generator (AWG, Keysight M8195A) and a 20-GHz oscilloscope (Tektronix DSA72004) to the RF probe card system and setting the input OOK-modulated signal at $20 \mathrm{Gbps}$ with a peak-to-peak voltage of $1 \mathrm{~V}$. The eye diagram shown in Figure $6 \mathrm{~b}$ obtained from the oscilloscope clearly indicates that the signal integrity is well preserved.

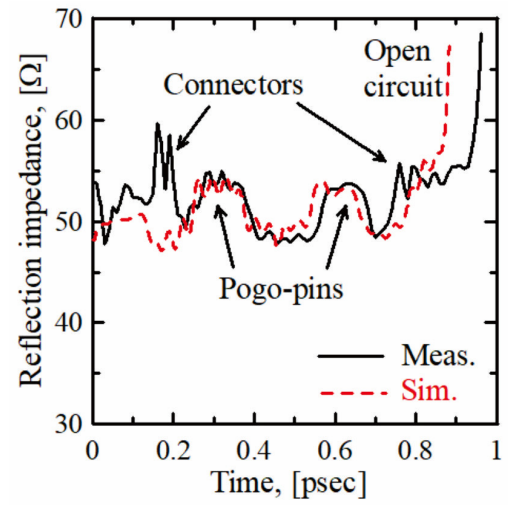

(a)

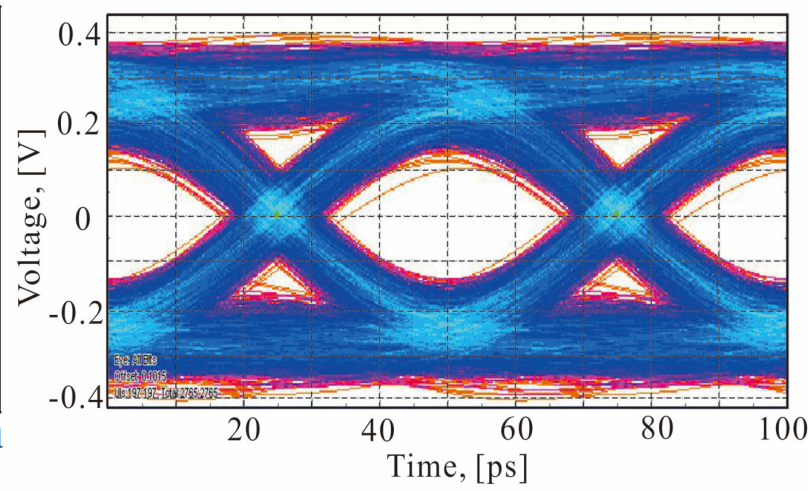

(b)

Figure 6. (a) TDR waveform converted from S-parameters and (b) measured eye diagram for 20 Gbps OOK modulation. 


\section{Design Improvement}

The prototype RF probe card possesses a sharp resonance near $28 \mathrm{GHz}$ that needs to be eliminated in the future as our eventual goal is to build 157-pin RF probe card that works up to at least $39 \mathrm{GHz}$. A series of HFSS simulations on the simplified probe card structure that is shown in Figure 7a suggests the resonance is triggered by the ground Pogo-pin arrangements. A pair of ground Pogo-pins connected to solid metal planes on both ends form a ground slot that resonates when the slot length becomes half wavelength. The half-wavelength resonance is verified from HFSS simulation results showing an E-field plot at the resonant frequency (Figure 7b). With the compressed pin length of $2.9 \mathrm{~mm}$, a resonance occurs near $28 \mathrm{GHz}$ causing significant reflection and radiation losses. In order to push the resonant frequency to beyond $40 \mathrm{GHz}$ as shown in Figure 7c, the RF probe card should be redesigned with the compressed Pogo-pin length shortened from 2.9 to $1.8 \mathrm{~mm}$.
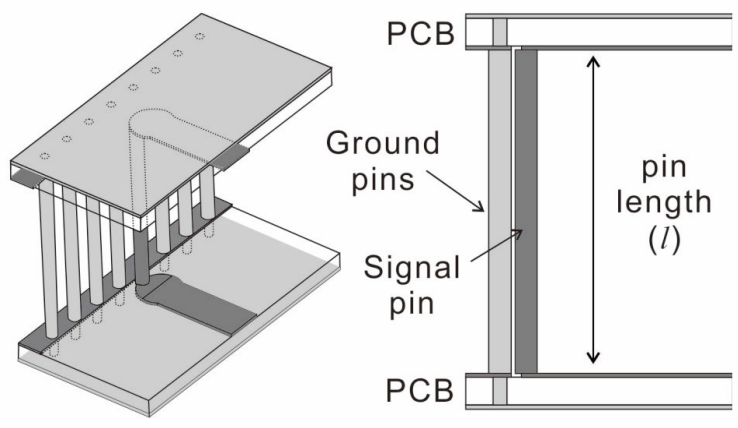

(a)

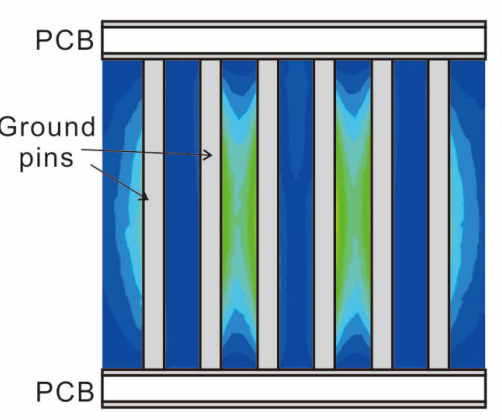

(b)

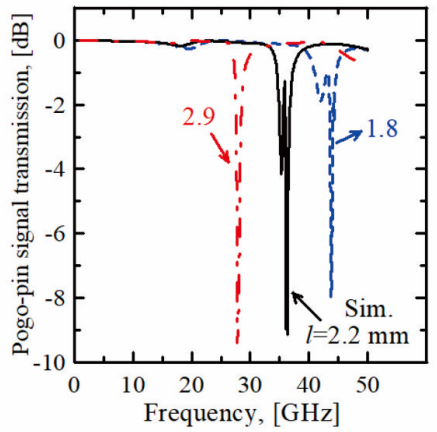

(c)

Figure 7. (a) Simplified Pogo-pin structure for HFSS analysis, (b) HFSS E-field plot at the resonance, and (c) simulated resonant frequency shifts for varying pin lengths.

\section{Conclusions}

A ceramic housing that holds an array of 157 pogo-pins is fabricated for the automatic testing of $29 / 39 \mathrm{GHz}$ on-wafer $5 \mathrm{G}$ phased-array SoCs. A prototype RF probe card is built by mounting the ceramic housing on a probe-card PCB that contains transmission lines to edge RF connectors. Measurements with Pogo-pins contacting a test PCB show successful signal delivery through the probe card with less than $3.4 \mathrm{~dB}$ of insertion loss at $25 \mathrm{GHz}$ indicating only a loss of $0.5 \mathrm{~dB}$ per each Pogo-pin connections. The manufacturing and testing of our prototype RF probe card demonstrates the flexibility of custom-made Pogo-pin arrays for future millimeter-wave integrated circuit testing. Further developments on the RF probe card with shorter Pogo-pins will continue in the future to cover the full 5G communication frequency range.

Author Contributions: Formal analysis, data curation, and original draft preparation, K.M.L.; housing fabrication and assembly, and technical guidance, J.H.O., M.S.K., and T.S.K.; supervision, M.K. All authors have read and agreed to the published version of the manuscript.

Funding: This research was supported by Samsung Electronics Co., Ltd. (No. 08405).

Conflicts of Interest: The authors declare no conflict of interest.

\section{References}

1. Rumiantsev, A.; Doerner, R. RF Probe Technology: History and Selected Topics. IEEE Microw. Mag. 2013, 14, 46-58. [CrossRef]

2. GGB Picoprobe Product List. Available online: https://www.ggb.com (accessed on 25 July 2021).

3. T-plus GSSG, GSGSG 67 GHz RF Probe. Available online: http://tplus-co.com/products01.html (accessed on 14 September 2021).

4. MPI Multi-contact probes. Available online: https://www.mpi-corporation.com/ast/mpi-rf-probes-accessories/titan-multicontact-probes (accessed on 25 July 2021).

5. Marinissen, E.J.; Fodor, F.; Wachter, B.D.; Kiesewetter, J.; Hill, E.; Smith, K. A Fully Automatic Test System for Characterizing Large-Array Fine-Pitch Micro-Bump Probe Card. In Proceedings of the CT-Asia, China-Taipei, Taiwan, 13 September 2017. 
6. Yokowo Connectors: Circuit Testing. Available online: https://www.yokowo.co.jp/english/project/connector/circuit.html (accessed on 25 July 2021).

7. Pasternack 40 GHz RF Probe. Available online: https://www.pasternack.com/rf-coaxial-gsg-probe-800-micron-pitch-40ghzcable-mount-2.92mm-pe2pb1004-p.aspx (accessed on 25 July 2021).

8. Tunaboylu, B. Electrical Characterization of Test Sockets with Novel Contactors. IEEE Trans. Dev. Mat. Reli. 2014, 14, 117-120. [CrossRef]

9. Yan, X.; Wang, Y.; Zhou, J.; Li, T.; Fan, J. A 20 GHz Landing Probe Design Based on Pogo-pins. In Proceedings of the EMC SI \& PI, Long Beach, CA, USA, 30 July 2018.

10. Dabrowiecki, K.; Gneiting, T.; Moreira, J. Evaluation of a spring probe card solution for 5G WLCSP application. In Proceedings of the IWLPC, San Jose, CA, USA, 22 October 2019.

11. Leeno Industry Inc RF Coaxial Pin. Available online: https:/ / leeno.com/products/fine-pitch-coaxial-probe-2 (accessed on 25 July 2021).

12. Fan, G.; Wang, Z.; Ren, H.; Liu, Y.; Fan, R. Dielectric dispersion of copper/rutile cermets: Dielectric resonance, relaxation, and plasma oscillation. Elsevier Scr. Mater. 2021, 190, 1-6. [CrossRef] 